\title{
Technical diagnostics of electrically operated valves at npp
}

\author{
Akhmetshin A.R*, Golomidov V.N*, Vildanov R.R** \\ *Kazan State Power Engineering University, Kazan, Republic of Tatarstan, Russia \\ *Smolensk branch "Smolenskatomtechenergo" JSC Atomtechenergo, Desnogorsk, Smolensk region, Russia \\ **Kazan State Power Engineering University, Kazan, Republic of Tatarstan, Russia
}

\begin{abstract}
Annotation. The article discusses the possibilities of technical diagnostics of electrically operated valves at nuclear power plants in order to increase their reliability and service life, as well as to achieve maximum operation efficiency of systems in nuclear power plants.
\end{abstract}

\section{Introduction}

Nuclear power plants (NPPs) in Russia throughout the entire period of operation have demonstrated reliable and safe operation in all areas of their activities. This has been confirmed by the results of regular inspections of both independent bodies (Rostekhnadzor) and international organizations (World Association of NPP Organizations).

Since 1999 , not a single serious safety violation has been recorded by Russian NPPs, classified above the first level on the INES International Scale (Nuclear Event Scale) [1].

Safety at a nuclear power plant is one of the most important properties to ensure the protection of both employees and the environment from radiation, both during normal operation and in case of possible accidents. An expedient level of safety is ensured by a system of physical obstacles erected in the path of radioactive substances, as well as measures aimed at maintaining their safety [2].

Secure operation of nuclear power plants largely depends on the operation of various devices and valves, which are a part of the main and auxiliary equipment of the plant.

\section{NPP technical diagnostics}

The purpose of technical diagnostics of electrically operated valves (EOV) at NPP is to increase its reliability and service life, as well as to achieve maximum efficiency of NPP systems operation by monitoring the readiness of electrically operated valves to perform their functions throughout the entire period of their operation, using the results of technical diagnostics to optimize the valves overhaul period, as well as to determine the volumes and types of repairs within the framework of the maintenance and repair strategy based on the technical condition, to process the results of technical diagnostics as an additional tool when performing work on monitoring, predicting and managing the resource of an electrically operated valves $[8,9,10]$.

Technical diagnostics of electrically operated valves allows to recognize the state of the EOV and determine the type of its technical condition, as well as to predict the technical condition for a given time interval. In addition, diagnostics allows you to identify places and determine the causes of failures or malfunctions [9].

These tasks are solved by analyzing the values of the measured electrical and mechanical diagnostic parameters based on the adopted diagnostic model, as well as by tracking the trend of the controlled parameters during the forecast. Fig. 1 and 2 show the diagnostic model of the EOV.

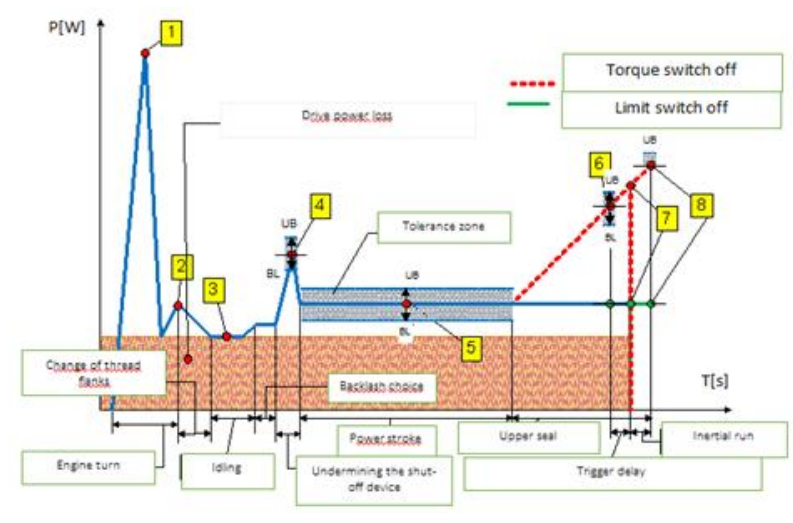

Fig.1.Diagnostic model EOV when opening the valve.

Characteristic points in the valve opening sequence diagram

Points:

No.1 - starting power;

No.2 - power of the spindle unloading start;

No.3 - idle power of the drive;

No.4 - power when undermining (wedging) the locking element;

No.5 - power when moving the locking element;

\footnotetext{
* Corresponding author: artur.akhmetshin.1991@mail.ru
} 
No.6 - power when the torque limiting clutch is triggered;

No.7 - maximum power before turning off the electric motor;

No.8 - maximum torque element in the end point.

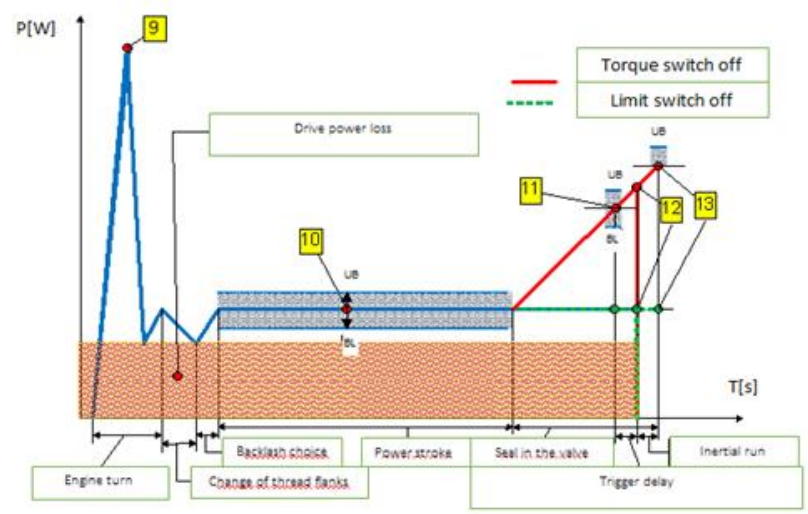

Fig.2.Diagnostic model EOV when closing the valve.

Characteristic points in the valve closing sequence diagram

Points:

No.9 - starting power;

No.10 - power when moving the locking element;

No.11 - power when the torque limiting clutch is triggered;

No.12 - maximum power before turning off the electric motor;

No.13 - maximum torque in the extreme position.

The definition of the relationship between torque and corrected active power is shown in fig. 3 .

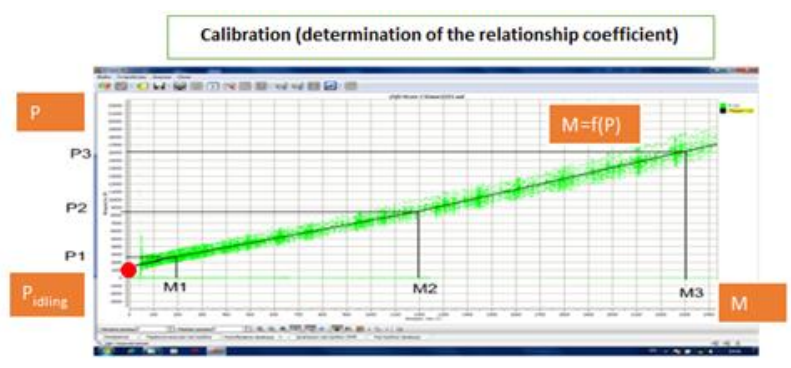

Fig.3.Determination of the relationship between torque and corrected active power.

While determining the relationship, the heating losses of cables and motor windings are taken into account, also mechanical losses on the drive, therefore, the drive can be used as a valve parameters sensor.

The nominal and limit values of the torques are determined analytically from the force design of the valve, data from passports or design documentation [9].

The torques (forces) at the characteristic points are calculated from the measured values of the corrected active power according to a predefined relationship:

$$
M=\frac{P_{c o r r}-P_{\text {idling }}}{C_{c}}
$$

The dependence is determined while testing electric drives on the bench or, if there is reliable data on the value of the couplings setting, in the drive certificates $[6,8,9]$.

Option 1. When using technical means that allow simultaneous recording of signals of active power and torque in the actuation cycle [9].

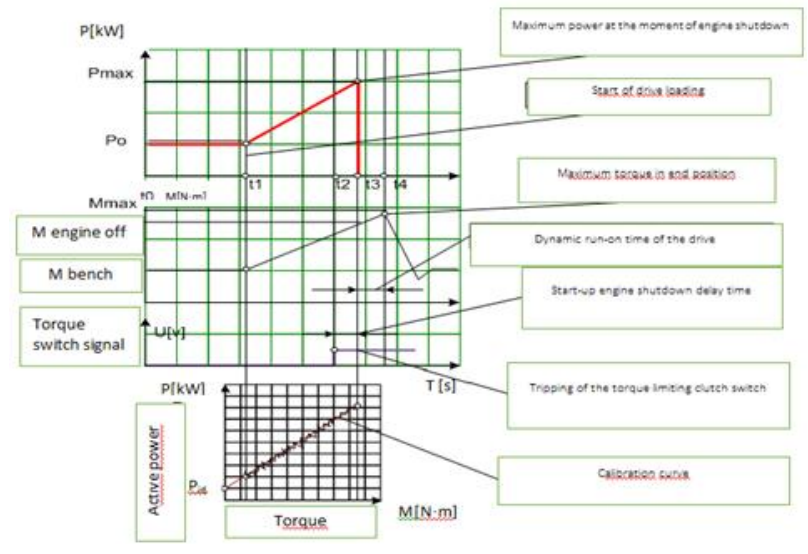

Fig.4.Graph of analysis of the measured active power and time intervals in the operation cycle.

t0 - end of the engine rotation;

t1 - start of the drive loading;

$\mathrm{t} 2$ - torque when the torque switch is triggered (TS);

t3--maximum value of active power before disconnection;

t4 - maximum torque at the end position.

The coefficient of the relationship between active power and torque is determined in the section tcaliber $=\mathrm{t} 2-\mathrm{t} 1$ from the load curve $M=f$ (Pcorr):

$$
C_{c}=\frac{P_{\text {corr }}-P_{\text {idling }}}{M}
$$

Option 2. When using technical means that do not have a digital channel for recording torque (Sprut-7).

Here, the loading of the stand braking device must be carried out in steps, taking and registering the readings of the active power and torque at each step. The dependence $\mathrm{M}=\mathrm{f}$ (Pcorr) is built according to the points corresponding to the number of loading steps [9].

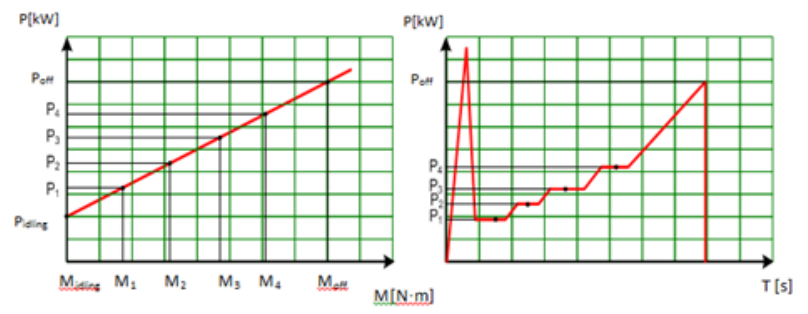

Fig.5.Graph of analysis of nominal and limiting values of torques in the operating cycle.

The $\mathrm{Cc}$ value is calculated from the linear dependence $\mathrm{M}$ $=\mathrm{f}$ (Pcorr) by the formula:

$$
P_{\text {corr }}=P_{\text {idling }}+C_{c} \cdot M
$$


Option 3: If you have reliable data on the amount of the electric drive [9].
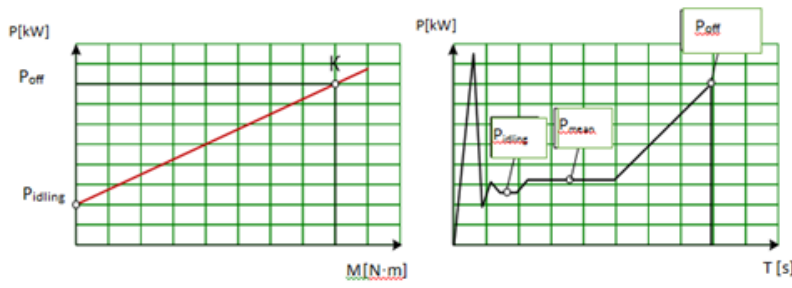

Fig.6.Graph of analysis of nominal and limiting values of torques in the actuation cycle with correction for real parameters of the working environment.

The Cc value is calculated from the linear dependence $\mathrm{M}$ $=\mathrm{f}$ (Pcorr) according to the formula:

$$
C_{c}=\frac{P_{o f f}-P_{i d l i n g}}{M_{o f f}}
$$

Where:

Pidling - corrected active idle power of the drive, $\mathrm{kW}$, measured during valve diagnostics;

Moff is the torque of the clutch setting specified in the passport, $\mathrm{N} \cdot \mathrm{m}$;

Poff is the corrected active power when the electric motor is turned off, $\mathrm{kW}$, measured during valve diagnostics.

Depending on the availability of the necessary data on valves and drives, as well as the capabilities of the equipment used, the nominal and limiting values of the parameters for assessing the technical condition of the EOV can be divided into three main options:

The first option is with limited information on the valve and without bench tests of the drive.

The technical condition (TC) is assessed by analyzing the measured active power and time intervals in the actuation cycle, as well as by imposing active power cyclograms on previous cyclograms in order to track changes.

The second option is if there is limited information on the valve, but with bench tests of the drive.

To evaluate the technical condition, in addition to option 1, the nominal and limit values of the torque in the operating cycle are used.

The third option is in the presence of all the information about the valve necessary for the power calculation and during the bench tests of the drive [9].

The assessment of the technical condition is carried out according to the parameters of option 2, but the values of these diagnostic parameters, in order to increase the resource of the valve, are corrected for the real parameters of the working environment. Torque values are calculated in accordance with the data and methods of power calculation ST TsKBA 002-2003, STTSKBA 086-2010 [3,4,5,7].

The diagnostic model adopted for EOV diagnostics is a set of diagnostic parameters (numerical values of torques and time intervals) at characteristic points on the graphs of recording current signals during its operation for opening and closing, for which the numerical nominal and boundary values [9].

The diagnostic method is based on determining the power and time characteristics of the valve by monitoring the active power consumed by the electric drive and torque in the actuation cycle.

Diagnostic parameters include:

1) recognition of the state of the technical system (determination of the type of technical condition) in conditions of limited information without disassembling the product;

2) search for the place of failure or malfunction;

3) the technical condition forecast.

The deviation of the monitored values of these parameters from the established ones indicates a change in the technical condition of the valves. By analyzing these deviations, the technical condition of the valve and the drive is recognized (their condition is assessed) and they are assigned to one of the types of technical condition.

The list of defects, which is determined based on the assessment obtained during diagnosis, includes the following:

1) the absence of phase;

2) time of the emergency valve to open;

3) emergency closing moment on the valve;

4) exceeding the stroke time to open the valve;

5) exceeding the stroke time to close the valve;

6) absence of connection of the control loop;

7) malfunction of the torque switch;

8) malfunction of the limit switch.

Today, diagnostics is a "tool" for monitoring the safe operation of nuclear power plants, and also solves such problems as:

1) ensuring the operational reliability of the controlled; NPP equipment due to early detection of its non-design states and operating modes;

2) assessment of the technical condition of the equipment, management of its service life, timely replacement of equipment that has developed a resource;

3 ) confirmation of the admissibility of changing the design conditions for the operation of the equipment (increasing the thermal power, lengthening the overhaul period);

4) management of the operating conditions of the monitored equipment based on the management of the aging of equipment elements and NPP systems, which means timely detection (diagnostics) of the equipment condition, repair and maintenance of operability at an acceptable level; as a result, the operating conditions of the equipment are softened, since they move away from the boundaries of hazardous operation;

5) optimization of costs for maintenance and repair in the context of an increase in the duration of overhaul cycles and optimization of the scope of technical and repair (including transfer of equipment for repairs based on technical condition)

Evaluation criteria of the technical condition of the EOV:

1) full stroke time;

2) smoothness of the working stroke;

3) the difference in transit time for opening / closing; 
4)the average torque of the working stroke on the running nut;

5)the response time of the valve during opening / closing;

6)the value of the torque force at the sealing of the closing element to close.

\section{Conclusion}

According to the results of the inspection of electric valve drives at Russian NPPs, which was carried out at the request of the Rosenergoatom concern, a satisfactory condition of the electrically operated valves was revealed at the Smolensk NPP (SNPP). Defects such as jamming of the valve due to incorrect setting of the torque clutch or the drive does not have enough effort to open the valve due to insufficient power of the electric motor, have not appeared in recent years at the SNPP.

Also, taking into account the positive experience of the Rostov NPP (RNPP) in commissioning with the diagnosis of EOV and the resulting zero failure of the EOV during testing, it can be concluded that the propaganda of technical diagnostics of electrically operated valves at Russian NPPs will allow early stages to identify defects and possible equipment failures.

The specialists of the Smolensk branch of Smolenskatomtechenergo have independently developed a complete, unparalleled in the industry, diagnostic support for electrically driven pipeline valves, including a diagnostic method; a specialized computer software complex ATE Valve Diagnostic DB for use by diagnostic departments of organizations when performing work on diagnostics of electrically driven pipeline valves; stand for testing, adjusting electric drives and determining their functional characteristics "ATZ TC-3000"; multifunctional device for measuring and recording electrical, mechanical and time parameters ATE SD-10 [9].

An important and urgent task of diagnosing the state of equipment during commissioning is the advanced detection of defects using various methods and techniques and the role of a quality barrier on the way of advancing defects and inconsistencies by the period when the power unit goes into operation [9].

Such a direction is the complex diagnostics of rotating units, the so-called multi-method, or the method of expert diagnostics, which performs the function of a "quality barrier", including:

the first barrier is advanced diagnostics of the technical condition of rotary equipment during acceptance tests at the manufacturer;

the second barrier is advanced diagnostics of the state during the installation period and at the first start-up of the equipment after installation.

Today, technical diagnostics of electrically driven industrial pipeline valves (IPV) is the most common type of diagnostics at NPPs, which is of practical interest due to its significant impact on NPP safety.

\section{References}

1. Personal site of JSC Rosenergoatom/Safety and ecology/Safety/NPP safety [Electronic resource]. URL: https://www.rosenergoatom.ru/

2. Personal site of JSC Atomenergomash/Media center/Information/Safety of nuclear power plants [Electronic resource]. URL: http://www.aemgroup.ru/

3. Garber D.Kh. Power fittings of high parameters. Moscow: Mechanical Engineering, 1968, 264 s.

4. D.F. Gurevich. Calculation of pipeline valves. L. Machine building. 1968, $888 \mathrm{~s}$.

5. Fittings for NPP equipment and pipelines. General technical requirements. OOT-87 M, 1987. [Electronic resource].URL: https://files.stroyinf.ru/Data2/1/4293829/42938293 39.pdf

6. A.Kh. Kizhner. Special valves for Nuclear Power Plants and their repair. M. Energoizdat. 1981, 104 s.

7. G.V. Arkadov and V.I. Pavlenko, B.M. Finkel. VVER diagnostic systems. Moscow. Energoatomizdat. 2010, 390 s.

8. Diagnostics of the technical condition of electrically operated valves/A.K. Adamenkov, I.N. Veselova//Electric stations. Issue 2. 2007, S. 53-56.

9. Method for diagnosing electrically operated valves with control of diagnostic parameters and experience of its application at Smolensk NPP. Dobrov//Nuclear stations. Maintenance and repair. Issue 1 (52). 2008, $145 \mathrm{~s}$.

10. Diagnostics of the state of electric motors. Method of spectral analysis of consumed current/V. Petukhov, V. Sokolov//News of electro-technology. Information and reference edition. Issue 1 (31). 2005, S. 50-52. 